\title{
Movilización Temprana Como Prevención Y Tratamiento Para La Debilidad Adquirida En La Unidad De Cuidados Intensivos En Pacientes En Ventilación Mecánica. Experiencia En Un Hospital De Segundo Nivel
}

\author{
Andrea Asenet Calixto Mejía, PT \\ Nancy Guadalupe Mendoza Martínez, PT \\ Universidad Politécnica de Santa Rosa de Jáuregui, \\ Lic. En Terapia Física, México \\ Orlando Rubén Pérez Nieto, MD \\ Hospital General de San Juan del Río de la \\ Secretaria de Salud del Estado de Querétaro, México \\ Miguel Ángel Martínez Camacho, PT
}

Universidad Autónoma de Querétaro, Licenciatura en Fisioterapia, México

Ernesto Deloya Tomas, MD

Beatriz Pérez Martínez, PT

Hospital General de San Juan del Río de la

Secretaria de Salud del Estado de Querétaro, México

Doi: 10.19044/esj.2018.v14n21p19 URL:http://dx.doi.org/10.19044/esj.2018.v14n21p19

\begin{abstract}
Introduction: The weakness acquired in the UCI is a condition that appears often in the critical patient, causing deficiencies in their physical and functional state. The early mobilization has proved to be safe and feasible demonstrating an improvement in the muscular strength and functionality of the patient during his stay in the ICU. Objectives: To describe the benefit of early mobilization in relation to muscle strength and functionality of critical patients upon discharge from the ICU. Material and methods: A retrospective, observational and descriptive study was conducted in the period from June to December of 2017, with a convenience sample of patients admitted to the ICU who were under mechanical ventilation and sedation, registration was obtained in the clinical files of muscle strength, functionality and mobility after the withdrawal of sedation and previous discharge of the patient, and the changes found were recorded. Results: A sample of 8 patients was obtained, of which $25 \%$ of the patients met the criterion of weakness
\end{abstract}


acquired in the ICU, in the IB it was observed that $100 \%$ of the patients obtained a severe dependency with a score between $21-60$ points and the IMS showed that $100 \%$ of the patients performed mobilization out of bed with or without assistance. A statistically significant difference was obtained with the Wilcoxon test: MRC $(p=0.012)$ and IB $(p=0.012)$. Conclusion: An early mobilization intervention favors the partial recovery of the complications of the stay in the ICU.

Keywords: Early mobilization, critical patient, intensive care unit, physical therapy, muscle strength

\section{Resumen}

Introducción: La debilidad adquirida en la UCI es una condición que se presenta con frecuencia en el paciente crítico, provocando deficiencias en su estado físico y funcional. La movilización temprana ha resultado ser segura y factible demostrando una mejoría en la fuerza muscular y funcionalidad del paciente durante su estancia en la UCI. Objetivos: Describir el beneficio de la movilización temprana en relación a la fuerza muscular y funcionalidad de los pacientes críticos al egreso de la UCI.Metodología: Estudio retrospectivo y descriptivo con muestreo no probabilístico de 8 pacientes que ingresaron a la UCI en el período junio a diciembre del 2017, en el cual se observó la fuerza muscular, funcionalidad y movilidad post sedación y previo al alta de la UCI a través de una revisión de expedientes clínicos que contaran con valoración e intervención por parte del área de Terapia Física. Resultados: Previo al alta de la UCI el $25 \%$ de los pacientes cumple con el criterio de debilidad adquirida, en el IB se observa que el $100 \%$ de los pacientes obtuvieron una dependencia severa con puntaje entre 21-60 puntos, por otro la IMS muestra que el $100 \%$ de los pacientes realizó movilización fuera de cama con o sin asistencia. Se obtuvo una diferencia estadísticamente significativa con la prueba de Wilcoxon: MRC $(\mathrm{p}=0.012)$ e IB $(\mathrm{p}=0.012)$. Conclusiones: Una intervención de movilización temprana favorece la recuperación parcial de las complicaciones de la estancia en la UCI.

Palabras Claves: Movilización temprana, paciente crítico, unidad de cuidados intensivos, terapia física, fuerza muscular

\section{Introduction}

La debilidad adquirida en la UCI (DA-UCI) y la disfunción neuromuscular asociada se detectan entre un $25-50 \%$ de los pacientes que requieren más de 5 días de ventilación mecánica (VM) invasiva, esto se asocia a la dificultad para el destete, estancia prolongada en la Unidad de Cuidados Intensivos UCI y un incremento de la morbimortalidad. La debilidad no solo 
se ve reflejada en los músculos de las extremidades, de igual manera la musculatura diafragmática produce una atrofia luego de 18 horas de iniciada la VM controlada, causando el retraso del destete ventilatorio, mientras que el mismo nivel de atrofia en los músculos esqueléticos de las extremidades se produce luego de las 96 horas de VM controlada. (Diaz et al., 2017).

Principalmente la inmovilidad ocasiona el síndrome de desacondiciona miento físico (SDC) en el paciente crítico causado por el reposo, desuso y la reducción de movilidad, lo cual trae como consecuencia un deterioro metabólico y sistémico del organismo, generando limitaciones, deficiencias y discapacidades. Los efectos de la debilidad adquirida en la UCI con frecuencia persisten o pueden dejar secuelas posterior al alta de la estadía en la UCI.(Cardona et al., 2014; Gómez, González, Olguin, \& Rodríguez, 2010; Via et al., 2013)

La Sociedad Americana de Tórax (ATS) define la debilidad adquirida en la UCI (DA-UCI) como un síndrome de debilidad de las extremidades generalizada que se desarrolla mientras el paciente está gravemente enfermo y para la cual no existe una explicación alternativa que no sea la propia enfermedad crítica. Este síndrome puede causar alteraciones en la función de los nervios ocasionando una polineuropatía del paciente crítico (PPC) o bien, una alteración en los músculos originando una miopatía del paciente crítico (MPC). (González, 2015).

Su etiología es multifactorial y se relaciona con diversos factores de riesgo importantes como son la edad, sepsis, la ventilación mecánica prolongada, la severidad de la enfermedad, el síndrome de respuesta inflamatoria sistémico (SIRS), la falla orgánica múltiple (FOM). Por otro lado el uso de bloqueadores neuromusculares, vasopresores, corticoesteroides y los opioides también se asocian a la DA-UCI. (Godoy, Vaz, Masotti, \& Napoli, 2015).

En los últimos años ha aumentado constantemente las intervenciones de rehabilitación física en pacientes críticos de las unidades de cuidados intensivos como resultado de la efectividad de la movilización temprana en pacientes de la UCI. Se ha demostrado que una adecuada prescripción de la terapia repercute de una mejora importante en la calidad de vida, la función física, la resistencia del musculo esquelético, respiratorio y periférico, la permanencia de estancia en la UCI y la duración de la ventilación mecánica en comparación con una atención estándar. (Connolly et al., 2015; Thomas et al., 2015).

La definición de movilización temprana es "la aplicación de la actividad física en los primeros dos a cinco días de enfermedad critica". La movilización temprana se caracteriza por un progreso jerárquico que abarca de las actividades funcionales de menor dificultad a actividades de mayor dificultad, estos comienzan de ejercicios pasivos, asistidos, activos y activos 
resistidos mientras los pacientes están en decúbito, posteriormente aumenta el grado de dificultad llevándolos a sedestación al borde de la cama, bipedestación, marcha sobre un mismo punto y finalmente hasta la deambulación, de forma segura, oportuna y eficaz desde el primer día. Se han desarrollado algoritmos clínicos de tratamiento basados en el estado de conciencia, estabilidad fisiológica, grado de desacondicionamiento y el nivel de participación activa de cada paciente. (Connolly et al., 2015; González, 2015).

En un estudio se observó que los días de ventilación mecánica promedio son de 14.4 con un tratamiento habitual en comparación con una intervención de movilización temprana (MT) donde se obtiene una reducción los días de VM con una media de 7.9 días, con una diferencia de 6.4 días $(\mathrm{p}=0.14)$, asimismo se observó una reducción de días de estancia a quienes se les aplico una intervención de MT, obteniendo una media de 17 días de estancia sin intervención de MT en comparación con una media de 10.7 con MT, con una diferencia de 6.3 días $(\mathrm{p}=0.17)$, demostrando así que la movilización y sedestación progresiva temprana influye de manera positiva. (Charry, Lozano, Rodríguez, Rodríguez, \& Mogollón, 2013).

Por otro lado el delirium en pacientes críticos es ahora reconocido como un importante problema de salud pública, que afecta hasta un $80 \%$ en pacientes de la UCI de adultos con ventilación mecánica. (Cruz \& Carrillo, 2015) Se ha reportado que en pacientes con intervención de terapia física y ocupacional tiene mejores resultados funcionales y menor duración de días de delirium. En un análisis realizado se demostró que el estado funcional independiente al alta hospitalaria en el grupo de intervención fue del $59 \%$ mientras que en el grupo control solo el $35 \%$ de los pacientes lo obtuvo. El grupo de intervención obtuvo una duración más corta de delirium con una media de 2 días, comparado con el grupo control que tuvo una media de 4 días. (Schweickert, Pohlman, \& Pohlman, 2009).

Muchos profesionales de la salud usan pruebas musculares manuales para evaluar la fuerza muscular, suelen usar la Medical Research Council (MRC), para cuantificar y comunicar sus conclusiones. Los procedimientos de pruebas musculares manuales y funcionales como esta escala son rápidos, seguros, simples de realizar, y no requieren equipo especializado. La MRC implica una prueba muscular manual, mediante un procedimiento para evaluar la fuerza de los grupos musculares, en función de la realización de un movimiento, en relación con la resistencia manual y la fuerza de gravedad. Se considera que un valor por debajo de los 48 puntos es debilidad adquirida en la UCI. (Nunes et al., 2016; Via et al., 2013) .

El índice de Barthel es un instrumento que mide la capacidad de una persona para realizar diez actividades de la vida diaria (AVD), consideradas como básicas, obteniéndose una estimación cuantitativa de su grado de 
independencia. Cada paciente se evalúa al principio y durante el tratamiento de rehabilitación, así como al momento del alta (máximo avance) ya que de esta manera, es posible determinar cuál es el estado funcional del paciente y cómo ha progresado hacia la independencia. (Barrero, García, \& Ojeda, 2005). La ICU Mobility Sacale (IMS) proporciona de una forma rápida y simple un método de cabecera para medir la movilidad del paciente críticamente enfermo. Evalúa su estado de movilidad mediante 11 puntos que van desde; puntaje 0 que es son movilidad, recostado, pasivo y ejercicios en la cama, a puntaje de 10 que es independiente y deambulación sin asistencia. (Tipping et al., 2016).

\section{Material y método}

Estudio de tipo retrospectivo, observacional y descriptivo en 8 pacientes en el periodo junio a diciembre del año 2017. Los criterios de inclusión fueron pacientes ingresados a la Unidad de Cuidados Intensivos del Hospital General San Juan del Río de la Secretaria de Salud del Estado de Querétaro, México, mayores de 18 años, que requirieron ventilación mecánica y sedación, sin contraindicaciones para realizar terapia física, con valoración por parte del área de terapia física y con registros reportados de fuerza muscular (MCR), funcionalidad (IB) y movilidad (IMS) posteriores al retiro de la sedación y previo egreso de los pacientes. El criterio de exclusión fue existencia de padecimientos neuromusculares previo ingreso a la UCI los criterios de eliminación fueron fallecimiento durante su hospitalización o traslado a otra unidad médica. No hubo pacientes excluidos ni eliminados del estudio.

Se realizó una revisión de los expedientes donde se adquirieron datos específicos: (Ficha de identificación; sexo, edad, diagnostico, signos vitales, fecha de ingreso, fecha de egreso, días de ventilación mecánica, días de estancia en la UCIA. Tratamiento farmacológico; sedación, analgesia, antibiótico, bloqueo neuromuscular y esteroides. Valoración por el área de Terapia Física: RASS, glasgow, REMS, fuerza muscular (MCR), independencia (IB) y movilidad (IMS) post sedación y previo al alta de la UCI.

Se recopiló la información obtenida a una base de datos utilizando el programa estadístico SPSS versión 21. Se realizó prueba de normalidad por medio Shapiro Wilk en las variables de fuerza muscular (MRC) e independencia (IB) encontrando que la muestra es de libre distribución no probabilística por lo cual se procedió a realizar la prueba de Wilcoxon.

\section{Resultados}

Se realizó un estudio con ocho pacientes $(n=8)$ que contaban con los criterios de inclusión. De los cuales el 75\% (6) fueron mujeres y el 25\% (2) hombres, con una edad promedio de $45.62 \pm 18.53$. El 37.5\% (3) cursaron con 
insuficiencia respiratoria y el 100\% (8) requirieron de ventilación mecánica invasiva. En todos los pacientes se utilizó sedación y analgesia. En el $87.5 \%$ (7) de los pacientes se utilizó propofol y en el 12.5\%(1) una combinación de propofol con midazolam, con una media de puntaje RASS de $-4.1 \pm 1.1$ al inicio de la ventilación. El 62.5\%(5) usó morfina como analgésico, $25.0 \%$ (2) tramadol y $12.5 \%$ (1) buprenorfina. Todos los pacientes tuvieron bloqueo neuromuscular con bromuro de vecuronio. (Gráfica 1. Grafica 2.)

Los pacientes tuvieron una media de estancia en UCIA de $14.00 \pm 5.09$ y una media de duración de ventilación mecánica invasiva de $7.12 \pm 3.27$. (Tabla 1.)

Posteriormente se evaluó fuerza muscular en 12 grupos musculares según los protocolos de la Medical Research Council (MRC) para la detección de debilidad adquirida en la UCI, índice de Barthel para cuantificar el grado de dependencia y la escala de movilidad en la UCI (IMS) después de la retirada de la sedación y previo al alta de la UCIA.

El puntaje de la MRC arrojó una media 27.25 \pm 7.75 al retirar sedación versus $50.12 \pm 4.67$ previo al alta de UCIA. Se encuentran 2 pacientes con un puntaje menor a 48 en la MRC previo al egreso de la UCIA, por lo tanto el $25 \%$ de los pacientes cumple con el criterio de debilidad adquirida en la UCI. Sin embargo cabe recalcar que el $100 \%$ de los pacientes tenían puntajes menores al retirar la sedación. (Tabla 2.)

Se obtuvo un índice de Barthel de $13.12 \pm 6.51$ tras la retirada de sedación en contra de $37.50 \pm 13.36$ previo al alta de UCIA. Se observa que el $100 \%$ de los pacientes al retirar la sedación tienen una dependencia total con puntaje $<20$ puntos versus el $100 \%$ una dependencia severa con puntaje entre 21-60 puntos al alta de UCIA. (Tabla 2.)

Por otro lado la IMS muestra un 75\% (6) de movilidad solo en cama y $25 \%$ (2) sin movilidad al retirar la sedación versus el $12.5 \%$ (1) mantuvo la bipedestación el $37.5 \%$ (3) camina con asistencia de 2 o más personas, $25 \%$ (2) camina con asistencia de una persona y un $25 \%$ (2) camina con asistencia de un auxiliar para la marcha previo al alta de UCI. (Tabla 3. Tabla 4.)

Se realizó prueba de normalidad por medio Shapiro Wilk en las variables MRC e independencia encontrando que la muestra es de libre distribución, posteriormente se realiza la prueba de Wilcoxon. En los ambos casos con una diferencia estadísticamente significativa: MRC $(\mathrm{p}=0.012)$ e independencia $(\mathrm{p}=0.012)$. (Gráfica 3, Gráfica 4). 
Tabla 1. Edad, dias de ventilación mecánica y dias de estancia en UCIA

\begin{tabular}{lllll} 
& Media & Desv. típ. & Mínimo & Máximo \\
\hline Edad & 45,625 & 18,5313 & 19,0 & 67,0 \\
Dias de Ventilación Mecánica & 7,125 & 3,2705 & 4,0 & 14,0 \\
\cline { 2 - 3 } & \multirow{2}{*}{14,000} & 5,0990 & 9,0 & 22,0 \\
\hline $\mathrm{n}=8$ & & & & HGSJR, 2017
\end{tabular}

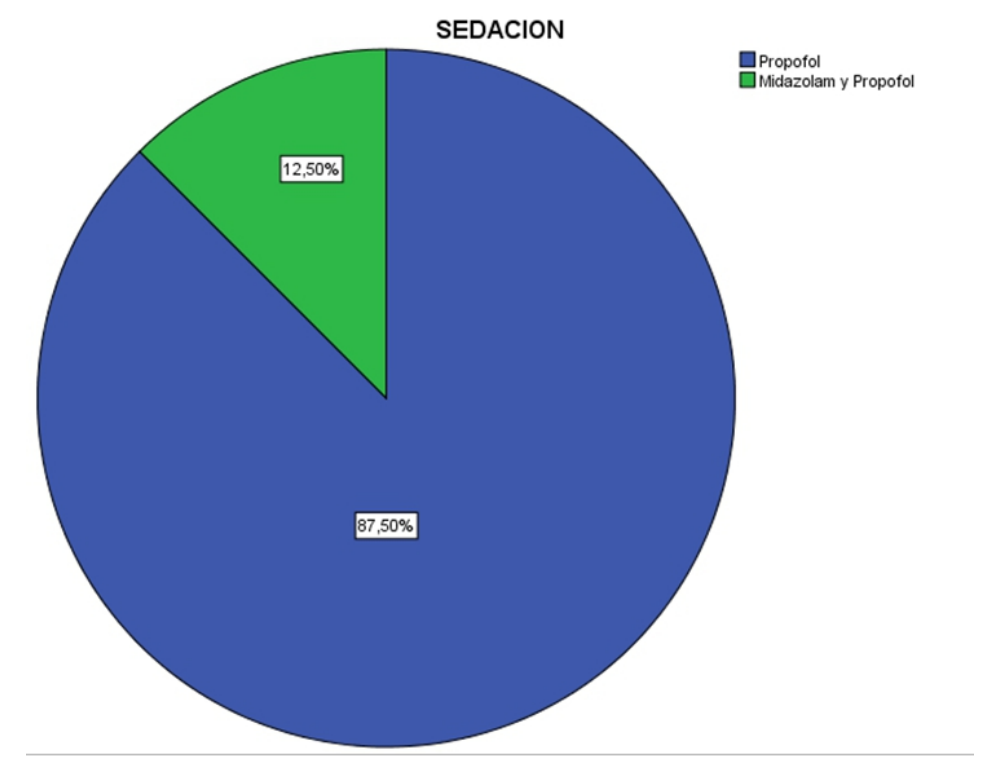

Gráfica 1. Porcentaje de Sedación

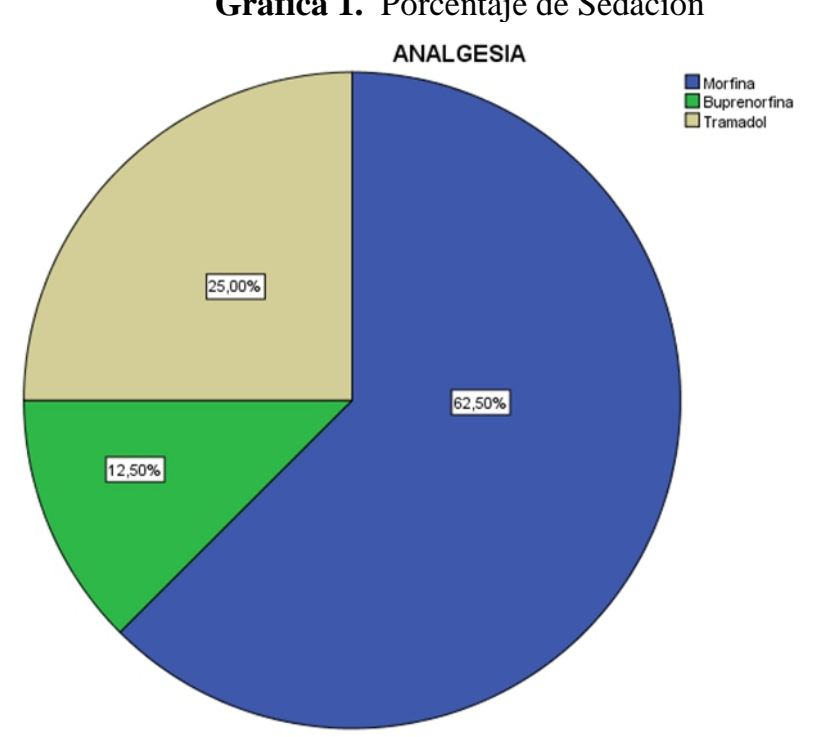

Gráfica 2. Porcentaje de Analgesia 
Tabla 2. Funcionalidad y fuerza en la UCIA

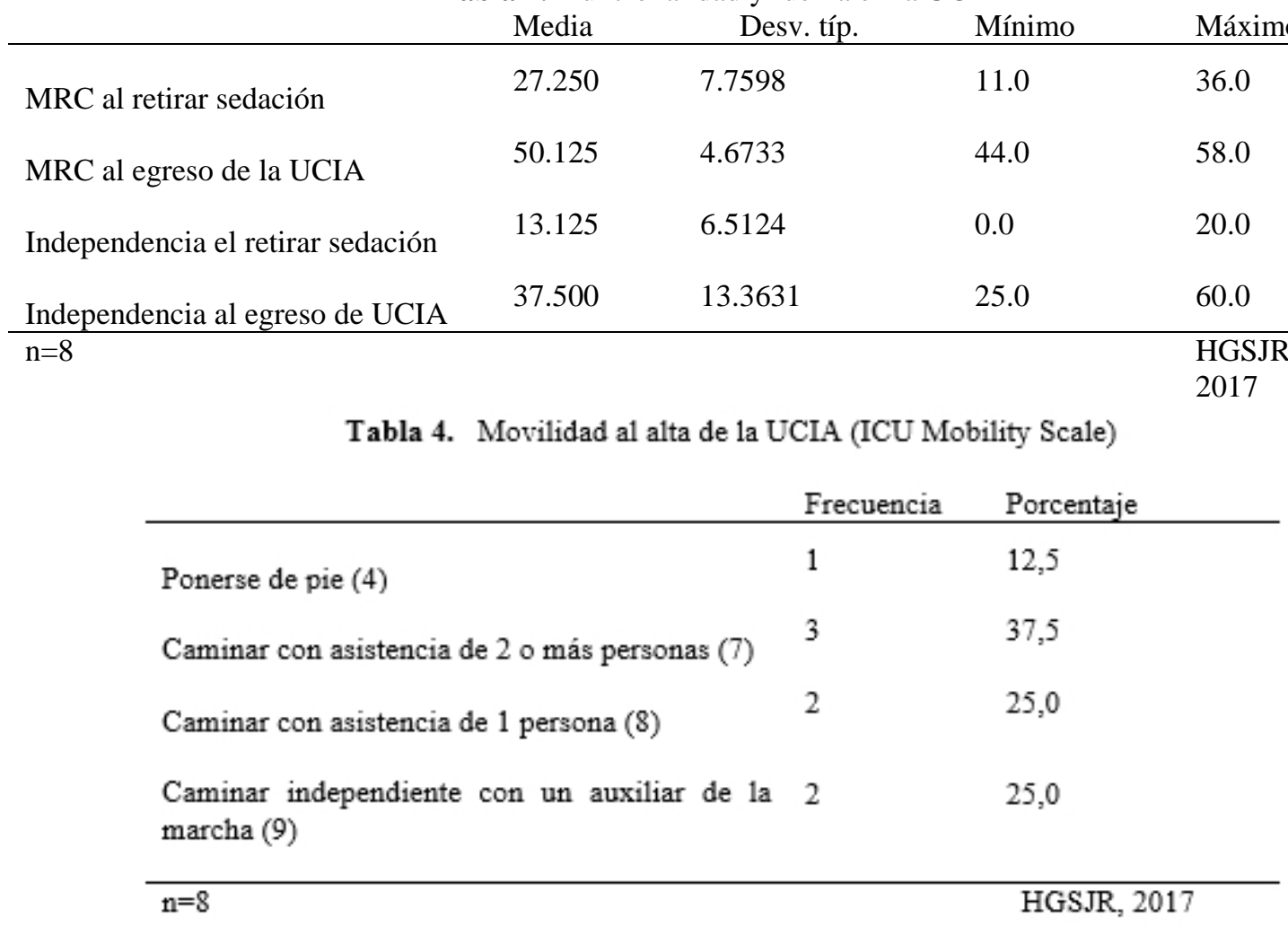

Tabla 3. Movilidad al retirar sedación (ICU Mobility Scale)

\begin{tabular}{llll} 
& & Frecuencia & Porcentaje \\
\hline Recostado en cama (0) & 2 & 25,0 \\
Sentado en la cama y ejercicio en cama (1) & 6 & 75,0 \\
\hline
\end{tabular}




\section{Medical Research Council}

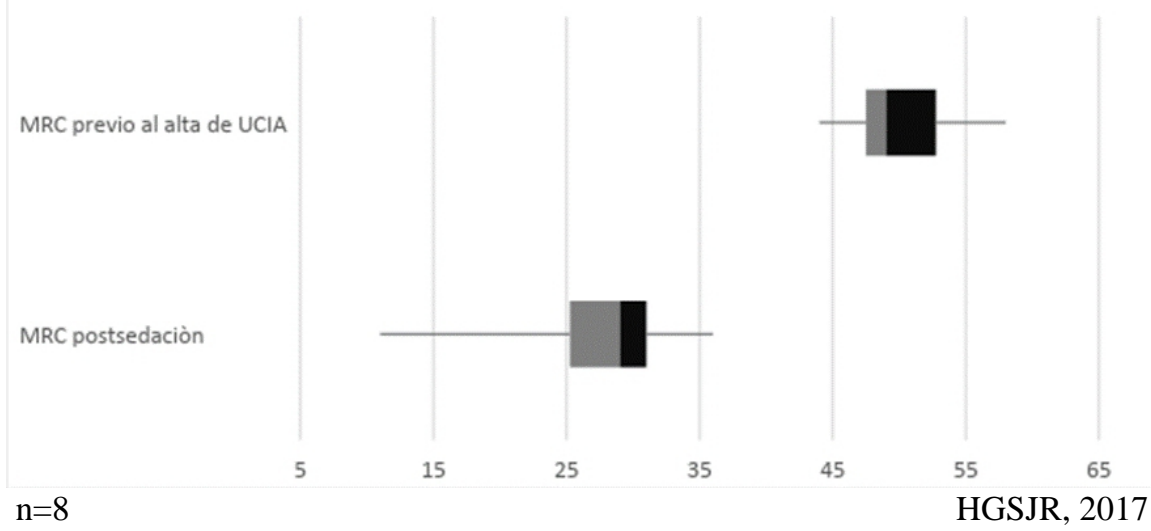

Gráfica 3. Resultados de fuerza muscular MRC (media), post-sedación versus al alta de UCIA

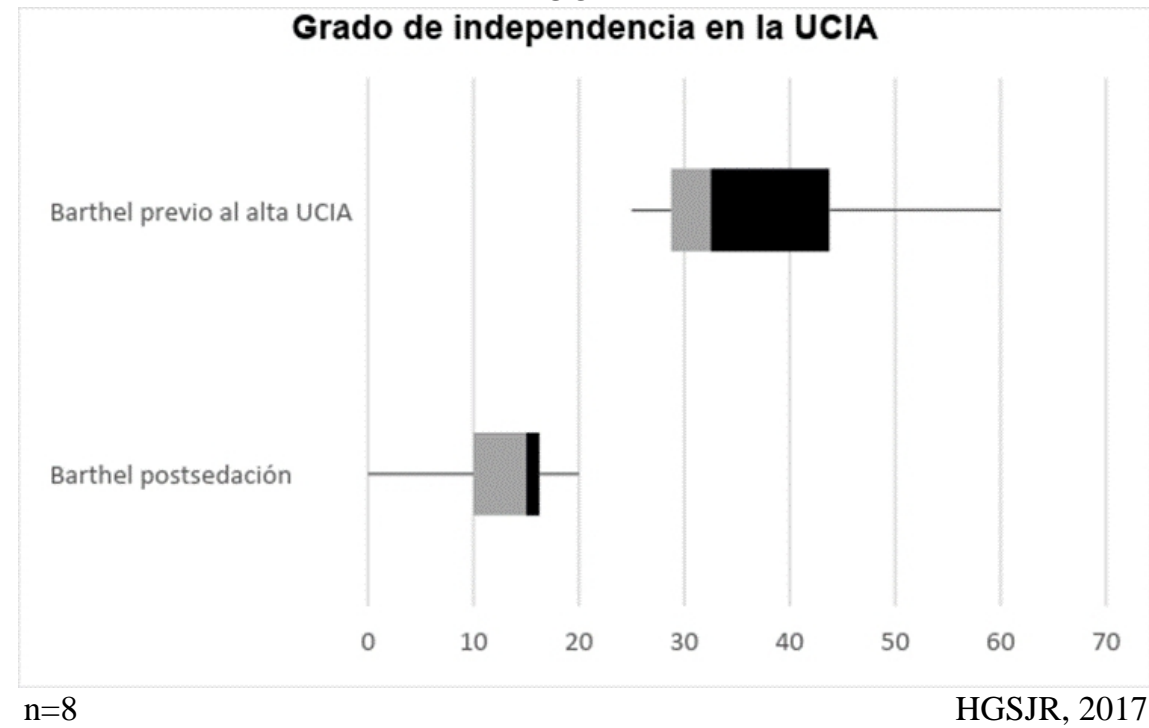

Gráfica 4. Resultados independencia IB, post-sedación versus al alta de UCIA

\section{Discusión}

Actualmente se reconoce la movilización temprana como una intervención viable y segura para los pacientes críticos que previene y/o mejora las secuelas funcionales derivadas de la debilidad muscular y el síndrome de desacondicionamiento así como disminuir los días de estancia hospitalaria, y los días de ventilación mecánica. (Connolly et al., 2015; Thomas et al., 2015; Tipping et al., 2016). Un hallazgo relevante en los valores de la fuerza muscular previos al alta de la UCI de acuerdo a la MRC el $25 \%$ de los pacientes obtuvo un puntaje de fuerza muscular menor a 48 puntos lo que corresponde a debilidad adquirida en la UCI, obteniendo resultados positivos siendo un porcentaje mínimo de acuerdo a estudios previos donde la 
DA-UCI se adquiere de entre un $25 \%$ hasta $50 \%$ en los pacientes críticos. (Diaz et al., 2017)

Por otro lado existió un importante aumento de movilidad detectando que el $100 \%$ de los pacientes realizaron movilizaciones fuera de cama previa al alta de la UCI, observando que el $37 \%$ realizo marcha con asistencia de 2 personas, $25 \%$ marcha con asistencia de una persona $25 \%$ marcha con auxiliar. Demostrando mejora significativa comparando estos resultados con la valoración inicial donde únicamente se muestra que el 25\% realiza movilidad en cama y $75 \%$ realiza movilidad en cama con sedestación asistida por camilla. Un estudio descriptivo realizado en una UCI en la ciudad de Cali, muestra las actividades de movilidad que se logran realizar al implementarse un protocolo de MT, el número de actividades fuera de cama fueron sentado en silla $19 \%$, posición bípeda $18 \%$ y deambulación $17 \%$, sin presentar ningún evento adverso, permitiendo concluir que la MT fue factible y segura. (Wilches et al., 2015).

En cuanto a los resultados de independencia (IB) se observó una leve mejoría identificando que previo al alta de UCIA el 100\% de los pacientes obtuvieron puntajes de 21 a 60 puntos considerado como dependencia severa comparado con los valores iniciales al retirar la sedación donde el 100\% obtuvieron puntajes $<20$ considerado como dependencia total. En un estudio similar, los pacientes con una implementación de la movilidad en la UCI obtuvieron una mejora funcional reflejada en los puntajes del IB que van de 45.9 en la admisión a la UCIA considerada como una dependencia severa a 85 puntos al alta de la UCIA considerada como una dependencia moderada (Fraser, Spiva, Forman, \& Hallen, 2015). De acuerdo a los resultados obtenidos del IB en ambos estudios concuerdan que existe una mejoría funcional previa al alta de la UCI sin lograr una independencia total. Sería prudente considerar el impacto que la terapia ocupacional tiene en el grado de dependencia funcional en los pacientes, se debe recalcar que el hospital donde se realizó el estudio no cuenta con terapeutas ocupacionales.

Debido a lo anterior se vuelve patente la importancia de la implementación de protocolos institucionales de movilización temprana en el área de UCIA realizado por personal calificado de fisioterapia y terapia ocupacional. De igual forma se considera necesario ampliar el tamaño de la muestra para obtener mejores resultados.

\section{Conclusión}

Los pacientes críticos no solo necesitan un tratamiento para resolutivo de la patología de base por el cual ingresan en la UCIA, se debe dar énfasis en los aspectos funcionales del mismo y de esta manera prevenir secuelas que causan discapacidad al alta. En esta investigación se puede observar el beneficio funcional obtenido por medio de la movilización temprana en la 
UCIA manifestándose como mejora en la fuerza muscular (MRC) grado de independencia (IB) y grado de movilidad (IMS). Existe una mejoría clínicamente significativa, sin embargo se requiere aumentar el tamaño de la muestra para poder realizar pruebas paramétricas y de esta manera los resultados tengan mayor peso estadístico. Es necesario la implementación de políticas y programas para favorecer la movilización en todas las áreas hospitalarias, de esta manera reducir los días de VM, disminuir la estancia hospitalaria y potencializar el beneficio funcional mejorando la calidad de vida de los pacientes previo al alta.

\section{References:}

1. Barrero, C., García, S., \& Ojeda, A. (2005). Índice de Barthel (IB): Un instrumento esencial para la evaluación funcional y la rehabilitación. Plasticidad Y Restauración ..., 4(1-2), 81-85. Retrieved from http://www.medigraphic.com/pdfs/plasticidad/prn2005/prn051_21.pdf

2. Cardona, E., González, A., Padilla, G., Páez, S., Alejo, L., \& Rodríguez, Y. (2014). Alteraciones Asociadas Al Desacondicionamiento Físico Del Paciente Crítico En La Unidad De Cuidado Intensivo. Revisión Sistemática. Revista Movimiento Científico, 8(1), 131-142. Retrieved from http://revistas.iberoamericana.edu.co/index.php/Rmcientifico/article/ view/742

3. Charry, D., Lozano, V., Rodríguez, Y., Rodríguez, C., \& Mogollón, P. (2013). Movilización temprana, duración de la ventilación mecánica y estancia en cuidados intensivos. Revista Facultad de Medicina, 61(4), 373-379.

Retrieved

from http://www.scopus.com/inward/record.url?eid=2-s2.084897075584\&partnerID=tZOtx3y1

4. Connolly, B., Salisbury, L., O’Neill, B., Geneen, L., Douiri, A., GrocottMPW., ... ERACIP, G. for the. (2015). Exercise rehabilitation following intensive care unit discharge for recovery from critical illness. Cochrane Database of Systematic Reviews, (6). https://doi.org/10.1002/14651858.CD008632.pub2.

5. Diaz, L., Dargains, N., Urrutia, J., Bratos, A., Percaz, M., Bueno, C., ... Settembrino, E. (2017). Debilidad adquirida en la unidad de cuidados intensivos. Incidencia, factores de riesgo y su asociación con la debilidad inspiratoria. Estudio de cohorte observacional. Revista Brasileira de Terapia Intensiva, 29(4). https://doi.org/10.5935/0103507X.20170063

6. Fraser, D., Spiva, L., Forman, W., \& Hallen, C. (2015). Original research: Implementation of an early mobility program in an ICU. 
American Journal of Nursing, 115(12), 49-58. https://doi.org/10.1097/01.NAJ.0000475292.27985.fc

7. Godoy, D., Vaz, L., Masotti, L., \& Napoli, M. (2015). Intensive Care Unit Acquired Weakness ( ICU - AW ): a brief and practical review. Reviews in Health Care, 6(1), 9-35. https://doi.org/10.7175/rhc.v6i1.1037

8. Gómez, M., González, V., Olguin, G., \& Rodríguez, H. (2010). Manejo de las secreciones pulmonares en el paciente crítico. $\begin{array}{lll}\text { Enfermeria Intensiva, 21(2), 74-82. } & \text { 21 }\end{array}$ https://doi.org/10.1016/j.enfi.2009.10.003

9. González, F. (2015). Movilidad Temprana en UCI. Equipo de KinesiologíaIntensiva Y Terapia Respiratoria Del Centro de Paciente Crítico Clínica INDISA.

10. Nunes, M., Hukuda, M., Favero, F., Oliveira, A., Voos, M., \& Caromano, F. (2016). Relationship between muscle strength and motor function in Duchenne muscular dystrophy. Arquivos de NeuroPsiquiatria, 74(7), 530-535. https://doi.org/10.1590/0004282X20160085

11. Schweickert, W., Pohlman, M., \& Pohlman, A. (2009). Early physical and occupational therapy in mechanically ventilated, critically ill patients: a randomised controlled trial. Lancet, 373, 1874-1882. https://doi.org/10.1016/S0140-6736(09)60658-9

12. Thomas, K., Wright, S. E., Watson, G., Baker, C., Stafford, V., Wade, C., ... Baudouin, S. V. (2015). Extra Physiotherapy in Critical Care (EPICC) Trial Protocol: a randomised controlled trial of intensive versus standard physical rehabilitation therapy in the critically ill. $B M J$ Open, 5(5), e008035. https://doi.org/10.1136/bmjopen-2015-008035

13. Tipping, C., Bailey, M., Bellomo, R., Berney, S., Buhr, H., Denehy, L., ... Hodgson, C. (2016). The ICU mobility scale has construct and predictive validity and is responsive: A multicenter observational study. Annals of the American Thoracic Society, 13(6), 887-893. https://doi.org/10.1513/AnnalsATS.201510-717OC

14. Via, G., Sanjuán, M., Menéndez, M., Corral, L., Martínez, G., \& Díaz, A. (2013). Evolución de la fuerza muscular en paciente críticos con ventilación mecanica invasiva. Enfermeria Intensiva, 24(4), 155-166. https://doi.org/10.1016/j.enfi.2013.09.001

15. Wilches, E., Muñoz, V., Girón, E., Ortega, D., Arias, M., Pelaez, G., \& Muñoz, D. (2015). Implementación de un programa de movilización temprana en pacientes críticos. Acta Colombiana de Cuidado Intensivo, 15(4), 287-292. https://doi.org/10.1016/j.acci.2015.08.003 